\title{
Computational Intelligence in Medical Decisions Making
}

\author{
Elizabeth Rakus-Andersson ${ }^{1}$ and Lakhmi C. Jain ${ }^{2}$ \\ ${ }^{1}$ School of Engineering, Department of Mathematics and Science, \\ Blekinge University of Technology \\ Karlskrona, Sweden \\ Elisabeth. Andersson@bth.se \\ ${ }^{2}$ School of Electrical and Information Engineering University of South Australia \\ Adelaide, Australia \\ Lakhmi .Jain@unisa.edu.au
}

\begin{abstract}
Computation intelligence paradigms including artificial neural networks, fuzzy systems, evolutionary computing techniques, intelligent agents and so on provide a basis for human like reasoning in medical systems. Approximate reasoning is one of the most effective fuzzy systems. The compositional rule of inference founded on the logical law modus ponens is furnished with a true conclusion, provided that the premises of the rule are true as well. Even though there exist different approaches to an implication, being the crucial part of the rule, we modify the early implication proposed in our practical model concerning a medical application. The approximate reasoning system presented in this work considers evaluation of a risk in the situation when physicians weigh necessity of the operation on a patient. The patient's clinical symptom levels, pathologically heightened, indicate the presence of a disease possible to recover by surgery. We wish to evaluate the extension of the operation danger by involving particularly designed fuzzy sets in the algorithm of approximate reasoning.
\end{abstract}

Keywords: Computational intelligence, approximate reasoning, compositional rule of inference, operation risk, symptom levels, parametric membership functions.

\section{Introduction}

Recent advances in computational intelligence techniques have offered tremendous opportunities to represent uncertain and imprecise knowledge in medical decision making. Artificial neural networks mimic the biological information processing mechanisms in a limited sense and help in fusing learning ability in decision making systems [1-11]. Fuzzy systems provide a means to transform computer programming to a sort of human reasoning system. Evolutionary computation involves a collection of algorithms based on the evolution of population towards a solution of a certain problem. Genetic algorithms, a part of evolutionary computing, are widely used in tasks such as optimization, automatic generation of artificial neural network architectures and so on. Multiagent systems are designed to act autonomously on behalf of the humans or users.---

E. Rakus-Andersson et al. (Eds.): Recent Advances in Decision Making, SCI 222, pp. 145-159. springerlink.com

(C) Springer-Verlag Berlin Heidelberg 2009 
The tremendous interest in the applications of computational intelligence in healthcare among researchers is evident by a number of publications in journals and conference proceedings. A decision support system for breast cancer detection using Bayesian networks is reported in [12]. The authors have used multiple images of each breast and demonstrated the merit of their approach in comparison to the single image system. The use of the personalized reasoning mechanism for an intelligent medical e-learning system on atheromatosis is reported in [13]. Atheromatosis of the aortic arch has been recognized as an important source of embolism, which is a frequent cause of stroke. A new classification technique of continuous EEG recordings based on a network of spiking neurons is presented in [14]. The merit of the proposed technique is demonstrated.

Decision support systems in wireless capsule endroscopy are revisited in [15]. The author has reported a study on pattern recognition system for texture characterisation and classification of capsule-endoscopic images. A decision support scheme for choosing antibiotic in open heart surgery is presented in [16]. Methods such as kernel density estimation, with market basket analysis and text analysis for compression are used in the implementation of decision support system.

Fuzzy rule induction and artificial immune systems in breast cancer familiarity profiling is reported in [17]. It is demonstrated that the biologically inspired data mining techniques are competitive tools in cancer research. Online analytical process methodology for assessing the risk of developing acute coronary syndromes is reported in [18]. It is demonstrated that the technique offers a more accurate risk assessment as it takes into account variable interaction. A fuzzy relational based medical diagnostic decision support system is reported in [19]. The authors claim that the system replicates closely a physician's perception of symptom-disease associations and his/her approximate reasoning for diagnosis. An online decision support system for diagnosing hematologic malignancies by flow cytometry immunophenotyping is reported in [20]. The system is expected to facilitate clinical diagnosis of hematologic disease. A multiagent based healthcare system is reported in [21]. The system is aimed to help telemedicine service, patient monitoring and diagnosis, emergency management, and so on.

Mobile collaboration framework for $u$-healthcare agent services is presented in [22]. The authors have demonstrated successfully the use of agents in healthcare applications. The use of mobile agents for diagnostic support in ubiquitous healthcare is reported in [23]. The authors have proposed a mobile agent for diagnostic support by using neuro-fuzzy algorithm for consultation report. The merit of the proposed system is demonstrated. Privacy-aware autonomous agent system for pervasive healthcare is reported in [24]. The authors have developed the system which takes into account contexural information such as the user's location and identify, the time of the day, the artifacts used, and the presence of colleagues to infer hospital worker's availability and privacy demands. A new paradigm for modelling illness in the human population is reported in [25]. The authors have reported a patient model using a mobile software agent. It is believed that the patients can investigate the effects of their life styles on their medical conditions. A hybrid intelligent medical diagnostic system using the fusion of fuzzy and evolutionary algorithms is reported in [26]. The 
system is designed to diagnose and prescribe treatment of blood gas disturbances and disorders. The diagnosis process is modelled using domain expert and existing literature.

A diagnostic support system for bladder tumor grading is reported in [27]. The authors have combined fuzzy cognitive maps with support vector machines to achieve better tumor malignancy classification. The proposed system presents better classification accuracy than the existing systems and thus able to make decisions with high diagnostic accuracy. A computer aided diabetes management system is reported in [28]. A good review of the computer aided diabetes education e-learning tool and an approach to therapy management is presented by the author. The Glucose-Insulin and Glycemic Index Web Simulator (GIGISim) tool deals with the patient needs. Rulebased assistance to Brain Tumor Diagnosis is presented in [29]. The authors have used a brain tumor database consisting of nuclear magnetic resonance spectroscopic signals. It is demonstrated that three spectral frequencies are sufficient to represent to diagnose human brain tumor. The design of a leaning environment for improving critical thinking skills in nursing domains is reported in [30]. The authors have presented the analysis of critical thinking. A learning system is proposed for facilitating decision making process. The system is in continuous improvement phase.

It is obvious from the above discussion that computational intelligence paradigms have become integral part of medical decision making. As a case study, we present the application of approximate reasoning in surgical decision making. The technique of approximate reasoning, earlier evolved by Zadeh [31,32] quickly found many adherents who differentiated the foundations of the theory. Especially, the changes concerned the implication IF...THEN...ELSE..., which constitutes an important factor of the reasoning system. In [33-35] we can trace the discussion revealing definitions of the implication generated by Kleene and Denies, Willmot, Mamdani and Assilian, Larsen, Gödel et al. The trials of inserting individually created operations on fuzzy sets discern the approaches mentioned above. Even the item of compositional rule of inference was debated from separate points of views [36-40]. We can mention the Yager conception [40] and the Sugeno design [33] as the most original modifications of the initial version of the rule.

For a practitioner an applicable meaning of approximate reasoning is essential, especially in technique and natural sciences where vagueness of input and output is often expected. Although some technical trials of applications are remarkable, it can happen coincidentally to counterpart the approximate reasoning in medicine. The only contribution in the topic, found by the author in [31], is a discussion of the model employing a pharmacological example.

Since members of surgical staff make decisions about operations on severely-ill patients with the highest care then we wish to support these verdicts by results coming from reasoning systems. We adopt Zadeh's approach to the rule [31, 32, 42], which is slightly modified by us and based on Lukasiewicz's definition of the fuzzy implication $[31,36,42]$. We still find this rule to be the most appealing for the reason of simply performed operations and clearly interpretable results. Then we build an own original apparatus accommodated to medical assumptions. Particular fuzzy sets that contain input data and output effects are designed in compliance with the physician's 
hint. The discussion about how to find the objective of reasoning, i.e. operation risk, is accomplished in Section 2. Fuzzy sets, taking place in the model, are furnished with appropriate membership degrees in Section 3. Section 4, added as a presentation of efficiency of the algorithm, reveals some risks in cancer surgery.

\section{Adoption of Approximate Reasoning to Operation Decisions}

For patients, who suffer from e.g. cancer, decisions concerning their operations are made with the highest thoughtfulness. In the later or the last stage of the disease the possibility to cure the patient totally of cancer by operating him/her for tumors is rather little. As a physician does not want the patient to run the risk to suffer even more after an unnecessary operation, he ought to judge thoroughly the consequences of the surgery.

We intend to involve approximate reasoning to support mathematically the extraction of a proper decision when discerning the operation danger. The most decisive clinical symptoms found in an individual patient will be taken into consideration to evaluate the risk.

Let us ponder a logical compound statement

$$
\begin{aligned}
& \operatorname{IF}(p \operatorname{AND}((\operatorname{IF} p \text { THEN } q) \operatorname{ELSE}(\operatorname{IF}(\operatorname{NOT} p) \\
& \text { THEN }(\text { NOT } q)))) \text { THEN } q
\end{aligned}
$$

whose primitive statements $p$ and $q$ are included in the equivalent form of (1) derived as

$$
p \wedge((p \rightarrow q) \wedge(\neg p \rightarrow \neg q)) \rightarrow q .
$$

The logical joint ELSE is interpreted in (2) as the conjunction $\wedge$ in compliance with the suggestions made by Lukasiewicz and Zadeh [31,36].

The logical statement (2) is a tautology, which can be easily confirmed by the method of truth tables. We also prove that thesis $q$ in (2) will become true if the premises $p$ and $(p \rightarrow q) \wedge(\neg p \rightarrow \neg q)$ constitute true statements as well. In order to accomplish the last proof we utilize the method of denying the truth of the thesis $q$. Let $v(p)$ and $v(q)$ denote the truth values of $p$ and $q$ according to the convention of binary logic. If, on behalf of the proof, we assume that the thesis $q$ is not true then $v(q)=0$. From the previous assumption $v((p \rightarrow q) \wedge \quad(\neg p \rightarrow \neg q))=1$ if $v(p \rightarrow q)=1$ and $v(\neg p \rightarrow \neg q)=1$. But $v(q)=0$, which suggests that $v(p)=0$ as well to warrant $v(p \rightarrow q)=1$. On the other hand we have already assumed that premise $p$ is true. As the suggestion $v(q)=0$ leads to the contradiction " $p$ is false" against " $p$ is true" then we will accept $v(q)=1$.

In accordance with the extended law modus ponens proposed by Zadeh [31, 32] we interpret (2) as a sentence

IF

$p^{`}$ (premise)

AND 
(IF $p$ THEN $q$ ) ELSE (IF (NOT $p$ ) THEN (NOT $q$ )) (premise)

THEN

$q^{\prime}$ (thesis)

provided that the semantic meaning of $p$ and $p^{`}$ ( $q$ and $q^{`}$ respectively) is very close.

Let $p$ be visualized by a fuzzy set $P$ in the universe $X$ and let $q$ be expressed by another fuzzy set $Q$ in the universe of discourse $Y$. Analogously, the fuzzy set $P^{`} \subset X$ constitutes a mathematical formalization of the primitive statement $p^{`}$ whereas $Q^{`} \subset Y$ replaces formally the sense of $q^{\prime}$. The modus ponens rule thus becomes

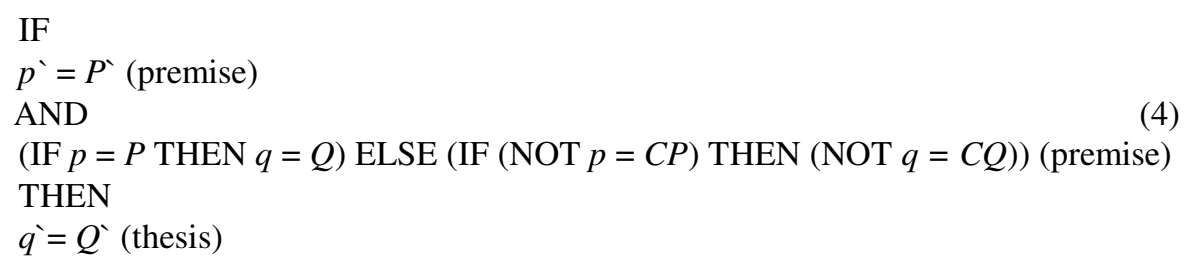

The sets $C P$ and $C Q$ are complements of $P$ and $Q$.

When making a feedback to the medical task previously outlined, we wish to use a technique of accommodating actual theoretical assertions to concrete formulations letting us evaluate the operation decision in some grades of risk.

Let $S$ denote a symptom possessing the most decisive power in the evaluation of the operation risk. We regard $S$ as either the complex qualitative symptom or the symptom whose intensity is assimilated with level codes. These codes, determined for both descriptions of $S$ 's complexion, form the universe $X=$ "symptom levels" = $\{1, \ldots, k, \ldots, n\}$. Let us assume that level 1 is associated with the slightly heightened symptom values whereas level $n$ indicates their critical status.

The statement $p$

$p^{`}=$ "symptom $S$ is found in patient on level $k$ "

is now addressed to a fuzzy set $P^{\prime}$ introduced by

$$
P^{`}=\frac{\mu_{P^{`}}(1)}{1}+\cdots+\frac{\mu_{P^{`}}(k)}{k}+\cdots+\frac{\mu_{P^{`}}(n)}{n} .
$$

The sentence $p$ built by

" $p=$ "rising levels of $S$ are essential for operation risk"

is dedicated to a fuzzy set $P$ given by

$$
P=\frac{\mu_{P}(1)}{1}+\cdots+\frac{\mu_{P}(k)}{k}+\cdots+\frac{\mu_{P}(n)}{n} .
$$

Another category of elements, constituting a content of the universe $Y$, is determined in the model as risk grades. We set risk grades in $Y=$ "operation risk grades" $=\left\{L_{0}=\right.$ "none", $L_{1}=$ "little", $L_{2}=$ "moderate", $L_{3}=$ "great", $L_{4}=$ "total" $\}$, on condition that $Y$ is experimentally restricted to five risk grades only. 
For sentence $q$

$q=$ "operation risk exists for patient"

a creation of a fuzzy set $Q$ is supported by

$$
Q=\frac{\mu_{Q}\left(L_{0}\right)}{L_{0}}+\frac{\mu_{Q}\left(L_{1}\right)}{L_{1}}+\frac{\mu_{Q}\left(L_{2}\right)}{L_{2}}+\frac{\mu_{Q}\left(L_{3}\right)}{L_{3}}+\frac{\mu_{Q}\left(L_{4}\right)}{L_{4}} .
$$

At last, we define $q^{`}$ containing the final risk judgment as a statement $q^{\prime}=$ "patient runs estimated risk of being operated",

where risk is graded by membership degrees of the corresponding fuzzy set $Q^{\prime}$ proposed as

$$
Q=\frac{\mu_{Q^{\prime}}\left(L_{0}\right)}{L_{0}}+\frac{\mu_{Q^{\prime}}\left(L_{1}\right)}{L_{1}}+\frac{\mu_{Q^{\prime}}\left(L_{2}\right)}{L_{2}}+\frac{\mu_{Q^{\prime}}\left(L_{3}\right)}{L_{3}}+\frac{\mu_{Q^{\prime}}\left(L_{4}\right)}{L_{4}} .
$$

In the next paragraph we accomplish the discussion about an apparatus providing us with membership degrees of sets (5)-(8).

Due to modus ponens rule (4) we set all decision data in the scheme

IF

"symptom $S$ is found in patient on level $k$ " $=P^{\prime}$ (premise)

\section{AND}

(IF "rising levels of $S$ are essential for operation risk" $=P$ THEN "operation risk exists for patient" $=Q$ ) ELSE (IF ("rising levels of $S$ are not essential for operation risk" $=C P$ THEN operation risk does not exists for patient $=C Q)$ (premise)

\section{THEN}

"patient runs estimated risk of being operated" $=Q$ (thesis)

In conformity with $[31,36,42]$ we first prognosticate a mathematical expression of the implication

(IF "rising levels of $S$ are essential for operation risk" $=P$ THEN "operation risk exists for patient" $=Q$ ) ELSE (IF ("rising levels of $S$ are not essential for operation risk" = $C P$ THEN operation risk does not exists for patient $=C Q)$

performed as matrix $R$. Even though several approaches to membership functions of implications were made $[31-33,35,36,38,42]$ we still feel attracted by the Lukasiewicz [36, 42] conception of fuzzy implication $R$ with a membership function derived as

$$
\begin{aligned}
& \mu_{R}\left(k, L_{l}\right)=1 \wedge\left(\left(1-\mu_{P}(k)\right)+\mu_{Q}\left(L_{i}\right)\right) \\
& \wedge\left(\mu_{P}(k)+\left(1-\mu_{Q}\left(L_{i}\right)\right)\right),
\end{aligned}
$$

$k=1, \ldots, n, l=0, \ldots, 4$, for all $x \in X$ and all $y \in Y$. 
The membership degrees of set $Q^{`}$ will be visualized after composing set $P^{`}$ with relation $R$ due to Zadeh's compositional rule [1]

$$
Q^{`}=P^{\prime} \circ R
$$

designated by the membership function

$$
\mu_{Q^{\prime}}\left(L_{l}\right)=\max _{k \in X}\left(\min \left(\mu_{P^{\cdot}}(k), \mu_{R}\left(k, L_{l}\right)\right)\right) \text {. }
$$

The comparisons of magnitudes of membership degrees in set $Q$ yield indications referring to judgments of the risk grades after consideration of symptom level $k$ verified in the patient.

As the operations of maximum and minimum have a tendency to filter the input data, which sometimes does not result in a clear-cut decision, then we will propose another set of composition operations in (10). In accordance with [43] we propose

$$
Q^{`}=P_{+}^{\prime} \circ R
$$

assisted by membership degrees

$$
\mu_{Q^{\prime}}\left(L_{l}\right)=\frac{\sum_{k=1}^{n} \mu_{P^{\cdot}}(k) \cdot \mu_{R}\left(k, L_{l}\right)}{\sum_{k=1}^{n} \mu_{R}\left(L_{l}\right)} .
$$

To be able to apply (13) we ought to prove that the value of the quotient $\mu_{Q}\left(L_{l}\right)$ is a number belonging to the interval $[0,1]$. To verify this we first notice that $\mu_{P^{\prime}}(k) \cdot \mu_{R}\left(k, L_{l}\right) \leq \mu_{R}\left(k, L_{l}\right)$ since both $\mu_{P^{\prime}}(k)$ and $\mu_{R}\left(k, L_{l}\right)$ are less than one for all $k$ and $l, k=1, \ldots, n, l=0, \ldots, 4$. This causes the value of a product to be lesser than the values of both factors. We thus conclude that the numerator is less than or equal to the denominator, which guarantees that the entire value of the quotient is a member of $[0,1]$; therefore it can be approved as a membership degree of $L_{l}$ coming from the support of $Q$.

We also notice that the sum placed in the denominator of the quotient never becomes equal to zero, since almost all risk grades will be designed as positive quantities. This assumption prohibits membership degrees of the risk grades from being undefined structures.

Values $\mu_{R}\left(k, L_{l}\right)$ are adaptable to be treated as weights of level importance assigned to a distinct risk. These, as the entries of matrix $R$ are invariants in the system promoting the same diagnostic model, contrary to information concerning different patients that is changeable. And, additionally, we can prove that operation (13) satisfies the criteria of OWA operators [43]. 


\section{Mathematical Design of Data Sets}

The decision model designed in Section 2 includes operations on fuzzy sets furnished with symbolically established membership degrees. In the current paragraph we put some life into theoretical symbols by assigning to them mathematical structures. The set $P^{`}$ a.k.a. (5) now gets assigned

$$
\begin{aligned}
& P^{`}=\frac{\mu_{P^{`}}(1)}{1}+\cdots+\frac{\mu_{P^{`}}(k)}{k}+\cdots+\frac{\mu_{P^{`}}(n)}{n} \\
& =\cdots+\frac{\frac{n-2}{n}}{k-2}+\frac{\frac{n-1}{n}}{k-1}+\frac{1}{k}+\frac{\frac{n-1}{n}}{k+1}+\frac{\frac{n-2}{n}}{k+2}+\cdots
\end{aligned}
$$

for the $k^{\text {th }}$ symptom level certified in the patient examined.

Another set $P$, concerning the same symptom levels in the support, is found by (6) and modified as

$$
\begin{aligned}
& P=\frac{\mu_{P}(1)}{1}+\cdots+\frac{\mu_{P}(k)}{k}+\cdots+\frac{\mu_{P}(n)}{n} \\
& =\frac{\frac{1}{n}}{1}+\cdots+\frac{\frac{k}{n}}{k}+\cdots+\frac{\frac{n}{n}}{n},
\end{aligned}
$$

due to the previously made assumptions, which suggest the tendency to ascending values of the membership degrees in $P$.

The set $Q$ is more sophisticated to design as a fuzzy set whose support consists of other fuzzy sets $L_{l}, l=0, \ldots, 4$, commonly defined in a symbolic risk reference set $Z=$ $[0,1]$. We also intend to determine the membership degrees of $Q$ as some characteristic quantities from $[0,1]$. Evaluation of these numbers is founded on a procedure involving a linguistic variable

"operation risk grades" $=\left\{L_{0}=\right.$ "none", $L_{1}=$ "little", $L_{2}=$ "moderate", $L_{3}=$ "great", $L_{4}=$ "total" $\}$,

experimentally restricted to five risk grades only.

We first fuzzify the expressions concerning the items of the list to continue further with their defuzzification in order to attach numerical equivalents to the words from the list. Each word assists now a fuzzy set $L_{l}, l=0,1,2,3,4$, whose constraint is grounded on an $s$-class mapping defined for $z$ in $Z=[0,1]$ as [44]

$$
\begin{aligned}
& \mu_{L_{l}}(z)=\mu_{L_{0}(l)}(z)=\left\{\begin{array}{l}
\operatorname{left}\left(\mu_{L_{0}(l)}(z)\right)= \\
\operatorname{right}\left(\mu_{L_{0}(l)}(z)\right)=
\end{array}\right. \\
& s\left(z, \alpha_{L_{0}}, \beta_{L_{0}}, \gamma_{L_{0}}, l \cdot h\right), \quad \text { for } z \leq \gamma_{L_{0}}, \\
& 1-s\left(z, \alpha_{L_{0}}+h, \beta_{L_{0}}+h, \gamma_{L_{0}}+h, l \cdot h\right) \text { for } z>\gamma_{L_{0}} .
\end{aligned}
$$

We clarify the fact that formulas of all membership functions are derived from only one predetermined subject defining $\mu_{L_{0}}(z)$. The equality $\mu_{L_{l}}(z)=\mu_{L_{0}(l)}(z)$ reveals 
that $\mu_{L_{l}}(z)$ is dependent on a parameter $l$ equal to level number $l, l=0, \ldots, 4$. The $h$ unit determines a distance between $\alpha_{L_{l}}$ and $\alpha_{L_{l+1}}$ (respectively $\beta_{L_{l}}$ and $\beta_{L_{l+1}}$ or $\gamma_{L_{l}}$ and $\gamma_{L_{l+1}}$ ) for symmetric functions $s$.

We prepare constraints for $L_{0}$, which are affected by $\alpha_{L_{0}}=-0.25, \beta_{L_{0}}=-0.125$ and $\gamma_{L_{0}}=0$ as

$$
\operatorname{left}\left(\mu_{L_{0}}(z)\right)=\left\{\begin{array}{l}
2\left(\frac{z-(-0.25)}{0-(-0.25)}\right)^{2} \\
\text { for }-0.25 \leq z<-0.125 \\
1-2\left(\frac{z-0}{0-(-0.25)}\right)^{2} \\
\text { for }-0.125 \leq z<0
\end{array}\right.
$$

and

$$
\operatorname{right}\left(\mu_{L_{0}}(z)\right)=\left\{\begin{array}{l}
1-2\left(\frac{z-0}{0.25-0}\right)^{2} \\
\text { for } 0 \leq z<0.125 \\
2\left(\frac{z-0.25}{0.25}\right)^{2} \\
\text { for } 0.125 \leq z<0.25 .
\end{array}\right.
$$

By inserting in (17) and (18) the current value $l, l=0, \ldots, 4$, and the distance $h$, casually determined as $h=0.25$, we obtain a formula of the left branch of $L_{l}$

$$
\begin{aligned}
& \operatorname{left}\left(\mu_{L_{l}}(z)\right)= \\
& \left\{\begin{array}{l}
2\left(\frac{z-(-0.25+l \cdot 0.25)}{0-(-0.25)}\right)^{2} \\
\text { for }-0.25+l \cdot 0.25 \leq z<-0.125+l \cdot 0.25, \\
1-2\left(\frac{z-(0+l \cdot 0.25)}{0-(-0.25)}\right)^{2} \\
\text { for }-0.125+l \cdot 0.25 \leq z<0+l \cdot 0.25,
\end{array}\right.
\end{aligned}
$$

and a function shaping its right branch

$$
\begin{aligned}
& \operatorname{right}\left(\mu_{L_{l}}(z)\right)= \\
& \left\{\begin{array}{l}
1-2\left(\frac{z-(0+l \cdot 0.25)}{0.25-0}\right)^{2} \\
\text { for } 0+l \cdot 0.25 \leq z<0.125+l \cdot 0.25, \\
2\left(\frac{z-(0.25+l \cdot 0.25)}{0.25-0}\right)^{2} \\
\text { for } 0.125+l \cdot 0.25 \leq z<0.25+l \cdot 0.25 .
\end{array}\right.
\end{aligned}
$$

Figure 1 collects plots of $L_{0}-L_{4}$ in conformity with different values of $l$ included in (19) and (20). 


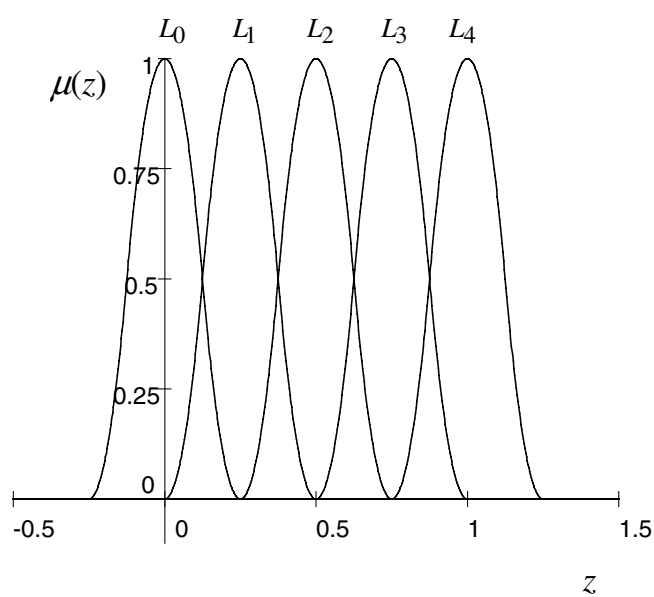

Fig. 1. The terms of "operation risk grades" as fuzzy sets $L_{0}-L_{4}$

Actually, we have an intension to emphasize the meaning of parametric nature of the $L_{l}$ membership functions, which deprives the model of many distinct formulas. Apart from this advantage we focus on generating the functions that represent elegant structures mathematically expressed.

In the process of defuzzification we consider only $z$-values for which the sets $L_{0}-L_{4}$ get the status of normal sets, i.e., $z=0, z=0.25, z=0.5, z=0.75$ and $z=1$. For these, another fuzzy set "numerical operation risk" is projected by developing its membership function in the form of

$$
\mu_{\text {"numerical operation risk" }}(z)=\left\{\begin{array}{l}
2\left(\frac{z-0}{1-0}\right)^{2} \\
\text { for } 0 \leq z<0.5 \\
1-2\left(\frac{z-1}{1-0}\right)^{2} \\
\text { for } 0.5 \leq z<1 .
\end{array}\right.
$$

Via the selected $z$-quantities above, we tie their membership degrees calculated by means of (21) to expressions from the list in order to establish relations between words and their numerical replacements. Therefore, the set $Q$ finally obtains a shape of

$$
Q=\frac{0}{L_{0}}+\frac{0.125}{L_{1}}+\frac{0.5}{L_{2}}+\frac{0.875}{L_{3}}+\frac{1}{L_{4}} .
$$

We now wish to demonstrate the action of approximate reasoning accustomed to the judgment of surgical risk.

\section{Risks Grades in Cancer Surgery}

In patients, who suffer from cancer as the recognized diagnosis, one of the symptoms, namely, $C R P$ ( $C$-reactive proteins) is carefully measured and discussed with a view to 
make a decision about accomplishing a successful operation. The heightened values of CRP (measured in milligrams per liter) are theoretically discerned in four levels stated as

$$
\begin{aligned}
& 1=\text { "almost normal" for } C R P<10, \\
& 2=\text { "heightened" if } 10 \leq C R P \leq 20, \\
& 3=\text { "very heightened" if } 20 \leq C R P \leq 25, \\
& 4=\text { "dangerously heightened" for } C R P>25 .
\end{aligned}
$$

Due to (15) set $P$ is expressed as

$$
P=\frac{0.25}{1}+\frac{0.5}{2}+\frac{0.75}{3}+\frac{1}{4}
$$

in $X=\{1, \ldots, 4\}$.

Suppose that an individual patient examined reveals the $C R P$-value to be 23. $C R P$ is thus classified in level 3 and set $P$ `characteristic of the patient is stated in the form of

$$
P `=\frac{0.5}{1}+\frac{0.75}{2}+\frac{1}{3}+\frac{0.75}{4} .
$$

according to (14).

The sets (23) and (22) together with

$$
\mathrm{C} P=\frac{0.75}{1}+\frac{0.5}{2}+\frac{0.25}{3}+\frac{0}{4}
$$

and

$$
\mathrm{C} Q=\frac{1}{L_{0}}+\frac{0.875}{L_{1}}+\frac{0.5}{L_{2}}+\frac{0.125}{L_{3}}+\frac{0}{L_{4}}
$$

generate matrix $R$ with the entries computed in compliance with (9). $R$ is expanded as a two-dimensional table

$$
\begin{aligned}
& \begin{array}{lllll}
L_{0} & L_{1} & L_{2} & L_{3} & L_{4}
\end{array}
\end{aligned}
$$

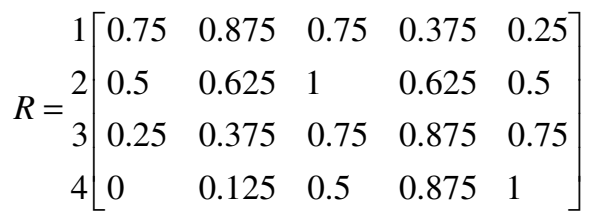

which, inserted in (10) for $P^{`}$ determined by (24), provides us with

$$
Q=\frac{0.5}{L_{0}}+\frac{0.625}{L_{1}}+\frac{0.75}{L_{2}}+\frac{0.875}{L_{3}}+\frac{0.75}{L_{4}} .
$$


By interpreting the meaning of (28) we understand that there exists a risk when considering an operation in patient whose $C R P$-index is evaluated on the third level. The most possible risk is evaluated as "great" according to the highest quantity of the membership degree. The total danger of accomplishing the surgical operation is evaluated as essential with the membership degree 0.75 .

Even the results of implementing (13) given as

$$
Q=\frac{0.66}{L_{0}}+\frac{0.69}{L_{1}}+\frac{0.75}{L_{2}}+\frac{0.795}{L_{3}}+\frac{0.725}{L_{4}} .
$$

fully confirm the risk extension judged by (28).

We hope that the classical model of approximate reasoning, modified by us and adapted to the problem of operation decision can constitute its complementary solution, especially when a decision of saving somebody's life via surgery is crucial.

\section{Conclusions}

We have presented an overview of the computational intelligence paradigms in medical decision making. As a case study, we have used approximated reasoning to introduce the initial interpretation of the system to approximate the operation risk concerning patients with rising values of a biological index. The formulas of membership degrees and membership functions have been expanded by applying a formal mathematical design. We expect that the study makes a contribution in the domain of mathematical models projected for medical applications.

In future works we wish to examine a model consisted of several symptoms that are divided in different numbers of levels. The symptoms should be included in the pattern simultaneously, which may expose some internal interactions among them. In other words, the operation risk will be a criterion that can employ many data factors. We count on finding some helpful remarks in [45] to implement an algorithm supporting the method newly planned.

\section{Acknowledgement}

This chapter is based on Professor Rakus Andersson's research paper accepted for presentation in 2009 IFSA World Congress, Lisbon, Portugal.

\section{References}

[1] Teodorescu, H.-N., Kandel, A., Jain, L.C. (eds.): Fuzzy and Neuro-fuzzy Systems in Medicine. CRC Press, USA (1999)

[2] Teodorescu, H.-N., Kandel, A., Jain, L.C. (eds.): Soft Computing in Human Related Sciences. CRC Press, USA (1999)

[3] Tonfoni, G., Jain, L.C. (eds.): Innovations in Decision support Systems, Advanced Knowledge International, ch. 8, 9 (2003) 
[4] Jain, A., Jain, A., Jain, S., Jain, L.C. (eds.): Artificial Intelligence Techniques in Breast Cancer Diagnosis and Prognosis. World Scientific, Singapore (2000)

[5] Teodorescu, H.-N., Jain, L.C. (eds.): Intelligent Systems and Technologies in Rehabilitation Engineering. CRC Press, USA (2001)

[6] Silverman, B., Jain, A., Ichalkaranje, A., Jain, L.C. (eds.): Intelligent Paradigms in Healthcare Enterprises. Springer, Heidelberg (2005)

[7] Husmeier, D., Dybowski, R., Roberts, S. (eds.): Probabilistic Modelling in Bioinformatics and Medical Informatics. In: Wu, X., Jain, L.C. (eds.) Advanced Information and Knowledge Processing Series. Springer, Heidelberg (2005)

[8] Ichalkaranje, N., Ichalkaranje, A., Jain, L.C. (eds.): Intelligent Paradigms for Assistive and Preventive Healthcare. Springer, Heidelberg (2006)

[9] Yoshida, H., Jain, A., Ichalkaranje, A., Jain, L.C., Ichalkaranje, N. (eds.): Advanced Computational Intelligence Paradigms in Healthcare 1. Springer, Heidelberg (2007)

[10] Vaidya, S., Jain, L.C., Yoshida, H. (eds.): Advanced Computational Intelligence Paradigms in Healthcare 2. Springer, Heidelberg (2008)

[11] Sardo, M., Vaidya, S., Jain, L.C. (eds.): Advanced Computational Intelligence Paradigms in Healthcare 3. Springer, Heidelberg (2008)

[12] Velikova, M.: A Decision support system for Breast Cancer Detection in Screening Programs. In: Proceedings of the 18th European Conference on Artificial Intelligence, pp. 658-662. IOS Press, Amsterdam (2008)

[13] Kabassi, K., et al.: Specifying the personalization reasoning mechanism for an intelligent medical e-learning system on Atheromatosis: An Empirical Study. Intelligent Decision Technologies-An International Journal 2(3), 179-190 (2008)

[14] Goel, P., et al.: On the Use of Spiking Neural Networks for EEG Classification. International Journal of Knowledge-Based and Intelligent Engineering Systems 12(4), 295-304 (2008)

[15] Kodogiannis, V.S.: Decision Support Systems in Wireless Capsule Endoscopy. Intelligent Decision Technologies-An International Journal 1(1-2), 17-32 (2007)

[16] Cerrito, P.B.: Choice of Antibiotic in Open Heart surgery. Intelligent Decision Technologies-An International Journal 1(1-2), 63-69 (2007)

[17] Menolascina, F., et al.: Fuzzy rule Induction and Artificial Immune Systems in Female Breast cancer Familiarity Profiling. International Journal of Hybrid Intelligent Systems 5(3), 161-165 (2008)

[18] Kostakis, H., et al.: A Computational Algorithm for the Risk assessment of Developing Acute Coronary Syndromes using Online Analytical Process Methodology. International Journal of Knowledge Engineering and Soft Data Paradigms 1(1), 85-99 (2009)

[19] Wagholikar, K.V., Deshpande, A.W.: Fuzzy Relation Based Modeling for Medical Diagnostic Decision Support: Case Studies. International Journal of Knowledge-Based and Intelligent Engineering Systems 12(5-6), 319-326 (2008)

[20] Qian, Y.-W., et al.: An On-line Decision Support System for Diagnosing Hematologic Malignancies by Flow Cytometry Immunophenotyping. International Journal of Medical Engineering and Informatics 1(1), 109-124

[21] Kang, E., et al.: Remote control multi-agent system for u-healthcare service. In: Nguyen, N.T., Grzech, A., Howlett, R.J., Jain, L.C. (eds.) KES-AMSTA 2007. LNCS (LNAI), vol. 4496, pp. 636-644. Springer, Heidelberg (2007)

[22] Jeong, C.-W., Kim, D.-H., Joo, S.-C.: Mobile collaboration framework for u-healthcare agent services and its application using pDAs. In: Nguyen, N.T., Grzech, A., Howlett, R.J., Jain, L.C. (eds.) KES-AMSTA 2007. LNCS (LNAI), vol. 4496, pp. 747-756. Springer, Heidelberg (2007) 
[23] Mateo, R.M.A., et al.: Mobile Agent Using Data Mining for Diagnostic Support in Ubiquitous Healthcare. In: Nguyen, N.T., Grzech, A., Howlett, R.J., Jain, L.C. (eds.) KESAMSTA 2007. LNCS (LNAI), vol. 4496, pp. 795-804. Springer, Heidelberg (2007)

[24] Tentori, M., et al.: Privacy-Aware Autonomous agents for Pervasive Healthcare. IEEE Intelligent Systems 21(6), 55-62 (2006)

[25] Nejad, S.G., et al.: An Agent-based Diabetic Patient Simulation. In: Nguyen, N.T., Jo, G.S., Howlett, R.J., Jain, L.C. (eds.) KES-AMSTA 2008. LNCS (LNAI), vol. 4953, pp. 832-841. Springer, Heidelberg (2008)

[26] Koutsojannis, C., Hatzillygeroudis, I.: Fuzzy-Evolutionary Synergism in an Intelligent Medical Diagnosis System. In: Gabrys, B., Howlett, R.J., Jain, L.C. (eds.) KES 2006. LNCS (LNAI), vol. 4252, pp. 1313-1322. Springer, Heidelberg (2006)

[27] Papageorgiou, E., et al.: Combining Fuzzy Cognitive Maps with Support Vector Machines for Bladder Tumor Grading. In: Gabrys, B., Howlett, R.J., Jain, L.C. (eds.) KES 2006. LNCS (LNAI), vol. 4251, pp. 515-523. Springer, Heidelberg (2006)

[28] Koleszynska, J.: GIGISim-The Intelligent Telehealth System: Computer Aided Diabetes Management - A New Review. In: Apolloni, B., Howlett, R.J., Jain, L. (eds.) KES 2007, Part I. LNCS (LNAI), vol. 4692, pp. 789-796. Springer, Heidelberg (2007)

[29] Nebot, A.: Rule-Based Assistance to Brain Tumor Diagnosis Using LR-FIR. In: Lovrek, I., Howlett, R.J., Jain, L.C. (eds.) KES 2008, Part II. LNCS (LNAI), vol. 5178, pp. 173180. Springer, Heidelberg (2008)

[30] Seta, K., et al.: Learning Environment for Improving Critical Thinking Skills in Nursing Domain. International Journal of Advanced Intelligence Paradigms 1(2) (2008)

[31] Zadeh, L.A.: Outline of a new approach to the analysis of complex systems and decision process. IEEE Transactions on Systems, Man, and Cybernetics MSC-3(1), 28-44 (1973)

[32] Zadeh, L.A.: A theory of approximate reasoning. Machine Intelligence 9, 149-174 (1979)

[33] Fuller, R.: On fuzzy reasoning schemes, http: //www.abo.fi/ rfuller/pgs97.pdf

[34] Hüllermeier, E.: Case based approximate reasoning. Springer, Heidelberg (2007)

[35] van den Broek, P.M.: Efficient algorithms for approximate reasoning. In: Proceedings of the 6th International Conference on Neural Information Processing, ICONIP 1999, Perth, Western Australia (1999)

[36] Baldwin, J.F., Pilsworth, B.W.: A model of fuzzy reasoning through multi-valued logic and set theory. Int. J. of Man-Machine Studies 11, 351-380 (1979)

[37] Cornelis, C., de Cock, M., Kerre, E.: Efficient approximate reasoning with positive and negative information. In: Negoita, M.G., Howlett, R.J., Jain, L.C. (eds.) KES 2004. LNCS (LNAI), vol. 3214, pp. 779-785. Springer, Heidelberg (2004)

[38] Gerla, G.: Fuzzy logic: mathematical tools for fuzzy reasoning. Kluwer, Dordrecht (2001)

[39] Mamdani, E.H., Assilian, S.: An experiment in linguistic synthesis with a fuzzy logic controller. Int. J. of Man-Machine Studies 7, 1-13 (1975)

[40] Yager, R.: An approach to inference in approximate reasoning. Int. J. of Man-Machine Studies 13, 323-338 (1980)

[41] Kilic, K., Sproule, B.A., Türksen, I.B., Naranjo, C.A.: A fuzzy system modeling algorithm for data analysis and approximate reasoning, https://research.sabanciuniv.edu/418/1/robotics_kemal.DOC.R

[42] Kacprzyk, J.: Fuzzy sets in system analysis. PWN, Warsaw (1986) 
[43] Rakus-Andersson, E.: Fuzzy and rough techniques in medical diagnosis and medication. Springer, Heidelberg (2007)

[44] Rakus-Andersson, E.: S-truncated functions and rough sets in approximation and classification of polygons. In: Proc. of Modeling Decisions for Artificial Intelligence - MDAI 2005, Tsukuba, CD-ROM, paper nr 049, Consejo Superior de Investigaciones Cientificas (2005)

[45] Wolkenhauer, O.: Fuzzy inference engines, composition and individual rule based on non-linear mappings, http: / /www. Systemsbiology.umist.ac.uk/ dokumente/dataengineering/infengines.pdf 
